\title{
Prospective Randomized Comparison between Single Session and Sequential Endoscopic and Laparoscopic Management of Concurrent Cholodocolithiasis and Cholecystolithiasis
}

\author{
Ibrahim H othman, ${ }^{1}$ MD; Taha A Esmail, ${ }^{1}$ MD; Hamdy Abdelhady, ${ }^{1} M D$; \\ Mohamed A Habllas; ${ }^{1}$ Medhat A Ghazy, ${ }^{2}$ MD.
}

1) General Surgery, Tanta University, Egypt.

2) Internal Medicine, Tanta University, Egypt.

The aim of this prospective randomized study is to compare single session endoscopic and laparoscopic management of concurrent cholodocolithiasis and cholecystolithiasis with the current practice where laparoscopic cholecystectomy is performed days after endoscopic stone extraction.

Patients and methods: This study was performed on 62 patients with concurrent cholodocolithiasis and cholecystolithiasis randomly categorized into 2 groups through a computer randomization program. Group I (30 patients) underwent endoscopic stone extraction and Laparoscopic cholecystectomy (LC) during the same session. This group was compared to 32 patients (Group II) who underwent endoscopic stone extraction and LC at least 3 days later.

Results: The characteristics of the two treatment groups, including baseline preoperative laboratory results showed no significant difference. In group I mean operative time was 97.3 \pm 17.9 minutes. In group II, collective mean operative time was $104 \pm 13.6$ minutes $(P=0.27)$. Mean hospitalization time was 2.33 \pm 1.45 in Group I and 2.94 \pm 1.29 days in Group II $(P=0.23)$. Mean Time to return to normal activity was $16.2 \pm 3.49$ and $15.9 \pm 4.31$ days $(p=0.85)$. Success rate of endoscopic procedures was $90 \%$ and $93.75 \%(P=0.67)$. Pancreatitis was observed in 2 patients in each group. Sphincterotomy related hemorrhage occurred in one patient in each group. One patient in group II experienced cholangitis. Total rates of endoscopic complications were $10 \%$ and $12.5 \%(P=1.000)$. Complications of ERCP were treated conservatively. No mortality was observed in both groups. Bile duct injury was not observed in this study. The overall LC related morbidity (including conversion) was 1/27 in group I and 2/30 in group II $(P=1.000)$. Level of direct bilirubin returned to normal values in the blood 9 days after procedure in both groups. Mean patient satisfaction score was $8.20 \pm 1.47$ versus $8.44 \pm 1.50$ $(p=0.66)$.

Conclusion: Endoscopic stone extraction and LC performed during the same session is feasible, safe and effective alternative to two-stage ERCP and LC for concurrent cholodocolithiasis and cholecystolithiasis. It has many advantages including avoiding a second procedure without increasing the length of operation, hospital stay or conversion rates to open procedure. We recommend more studies to be done on this subject before its routine recommendation in surgical practice.

Key words: Single session, sequential, endoscopic, laparoscopic, concurrent cholodocolithiasis and cholecystolithiasis.

Abbreviations: CBD: Common bile duct, ERCP: Endoscopic retrograde cholangiopancreatography, ES: Endoscopic sphincterotomy, FET: Fisher's exact test, LC: Laparoscopic cholecystectomy, LCBDE: laparoscopic common bile duct exploration. 


\section{Introduction:}

The incidence of gallstones varies from $6 \%$ to $10 \%$ in adult population. Their treatment depends on clinical presentation. The "gold standard" treatment for cholecystolithiasis is laparoscopic cholecystectomy, whereas the "gold standard" treatment for isolated CBD stones, especially in cholecystectomized patients, is endoscopic clearance. ${ }^{1}$ On the other hand, when gallstones and CBD stones are present concurrently, the treatment is a challenge. A consensus on optimal management does not exist. Several approaches are used, all having their proponents, such as open surgery, laparoscopy, and laparoendoscopic treatments, either sequential or simultaneous.

Synchronous CBD stones exist in 10\%$18 \%$ of patients undergoing laparoscopic cholecystectomy (LC) for gallbladder stones. ${ }^{2,3}$ They should be treated even if asymptomatic. ${ }^{4}$ Prior to the development of minimally invasive surgery, when the surgical approach to CBD stones consisted of open choledocholithotomy, there was considerable morbidity (11-14 \%) and even mortality (0.6-1 \%). 5 After the advent of endoscopic and laparoscopic techniques, CBD stones are removed preoperatively by endoscopy, followed by LC. ${ }^{6}$ With refinements in laparoscopic techniques and experience many centers have started performing laparoscopic common bile duct exploration with acceptable outcome. ${ }^{7}$

As the debate on the optimal therapeutic choice is ongoing, surgeons are often bewildered by the variety of options which have emerged in the minimally invasive era. Early LC after endoscopic CBD stone extraction is currently considered a good algorithm. ${ }^{8-10}$ LC combined with endoscopic clearance of CBD stones is an appropriate option. There are only few randomized trials available comparing the single stage with sequential management (ERCP followed by LC) of patients with concomitant gallstones and CBD stones. ${ }^{11,12}$

In the present prospective randomized study, we compared single session endoscopic and laparoscopic management of concurrent cholodocolithiasis and cholecystolithiasis with the current practice where laparoscopic cholecystectomy is performed days after endoscopic stone extraction.

\section{Patients and methods:}

This study was performed in the General Surgery and Internal medicine Departments, Tanta University Hospitals, Egypt during the period from October 2012 to June 2015 on 62 patients with gallstones and CBD stones. Full explanation of procedures; possible complications and patient consent were assured before inclusion in the research. The study protocol was approved by the Ethics Committees Tanta University Hospitals.

Patients were randomly categorized into 2 groups through a computer randomization program (www.randomization.com). Group I included 30 patients who underwent endoscopic stone extraction and LC during the same session. This group was compared to 32 patients who underwent endoscopic stone extraction and LC at least 3 days later. (Group II).

Exclusion criteria: these patients were excluded from our study

- Bleeding tendency (INR >1.7)

- Acute pancereatitis.

- Acute cholangitis.

- Stone CBD >20mm.

- Platelet count $<50,000$

- Bilio-enteric fistula.

- Pancereaticobiliary malignancy.

- Previous surgery altering location of the papilla.

All patients underwent detailed medical history, clinical examination, in addition to routine hematological, biochemical and radiological investigations for the diagnosis. Patients were hospitalized on the day of surgery.

In group I, general anesthesia with endotracheal intubation was usedin all patients. ERCP was performed with the patients in the prone position. A duodenoscope was inserted into the duodenal second segment via the mouth. A cholangiogram was carried out using C-arm X-ray and an endoscopic sphincterotomy was performed to extract the 


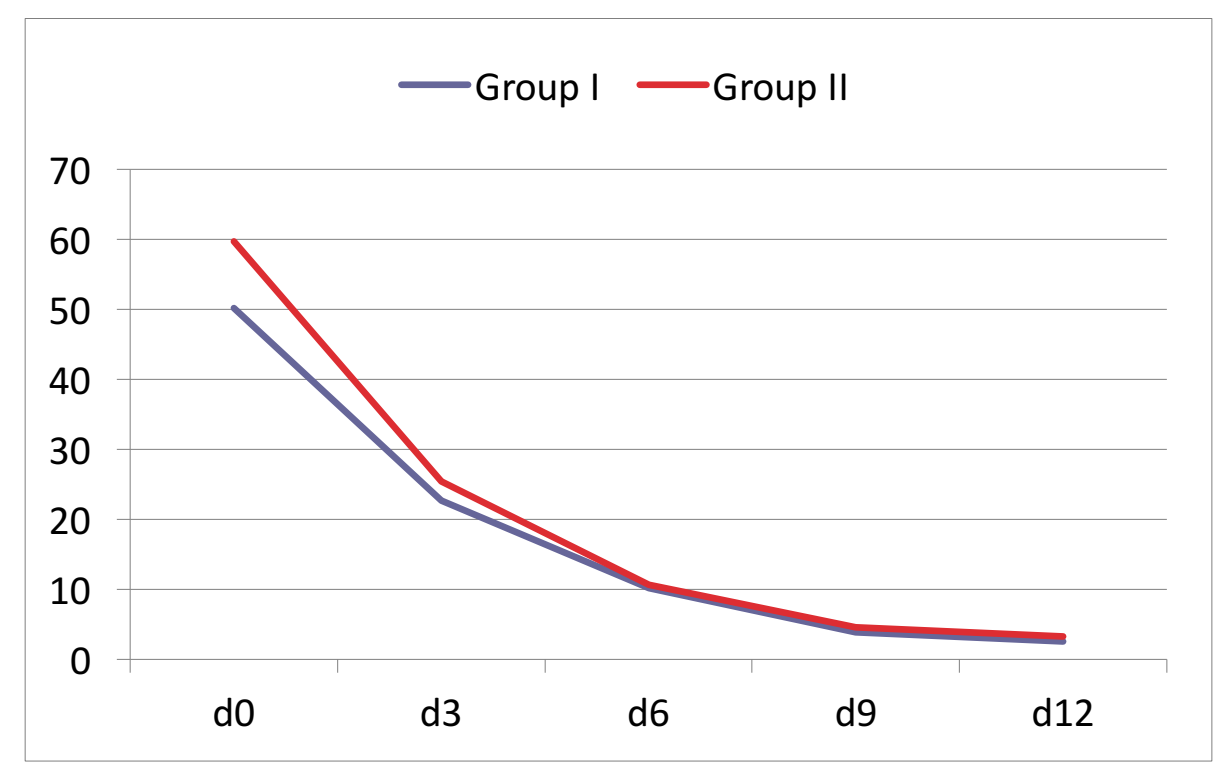

Figure (1): Level of direct bilirubin returned to normal values in the blood 9 days after procedure in both groups. Vertical axis: Direct bilirubin ( $\mu \mathrm{mol} / \mathrm{L})$ Horizontal axis: days after procedure.

Table (1): The demographic characteristics and baseline preoperative laboratory results of patients.

\begin{tabular}{|l|l|l|l|}
\hline & \multicolumn{1}{|c|}{ Group I } & \multicolumn{1}{c|}{ Group II } & \multicolumn{1}{c|}{ p } \\
\hline Age (year) & $49.2 \pm 12.3$ & $45.5 \pm 15.5$ & 0.47 \\
\hline Sex (male/female) & $7 / 23$ & $9 / 23$ & 0.77 \\
\hline ASA (I/II/III) & $19 / 7 / 4$ & $22 / 6 / 3$ & \\
\hline Diameter of CBD (mm) & $12.5 \pm 2.07$ & $12.8 \pm 2.32$ & 0.66 \\
\hline Maximal diameter CBD stone (mm) & $7.00 \pm 1.46$ & $6.81 \pm 1.22$ & 0.70 \\
\hline Direct bilirubin $(\mu \mathrm{mol} / \mathrm{L})$ & $59.6 \pm 19.4$ & $50.1 \pm 19.5$ & 0.19 \\
\hline Aspartate aminotransferase(U/L) & $145 \pm 40.3$ & $133 \pm 45.4$ & 0.45 \\
\hline Alanine aminotransferase (U/L) & $79 \pm 40.2$ & $83 \pm 44.0$ & 0.80 \\
\hline Alkaline phosphatase (U/L) & $62 . \pm 25.7$ & $57 \pm 27.1$ & 0.58 \\
\hline
\end{tabular}

CBD stones. The stones were removed by basket or balloon catheter. Stones larger than $10 \mathrm{~mm}$ were removed using a mechanical lithotripter. Following ERCP, care was taken to remove all the gas from the stomach to facilitate LC. The patients were then placed in supine position with a steep head-up and left tilt. Classic 4 port LC was performed. A sub hepatic drain was positioned if there was concern about the anatomy of the cystic duct and a danger of bleeding.

In group II, patients underwent endoscopic stone extraction with intravenous general anesthesia. If there were no complications related to ERCP, LC was performed three days later. Otherwise, LC was delayed until the ERCP related complications were successfully treated.

Patients were allowed to drink 6 hours after recovery. Analgesia was given in the form of $100 \mathrm{mg}$ Pethedine on recovery and another $100 \mathrm{mg}$ Pethedine intramuscularly after 12 hours if the patient complains of pain. Postoperative pain was measured 6 hours after surgery using pain visual analog scale.

Patients were investigated 12 hours after, by abdominal U/S, MRCP, complete blood count, liver function panel, serum amylase, lipase

Group I Patients were discharged from 
Table (2): Outcome of LC and endoscopic procedures.

\begin{tabular}{|l|l|l|l|}
\hline & \multicolumn{1}{|c|}{ Group I } & \multicolumn{1}{c|}{ Group II } & \multicolumn{1}{c|}{ p } \\
\hline Mean operation time (minutes) & $97.3 \pm 17.9$ & $104 \pm 13.6$ & 0.27 \\
\hline Mean hospitalization time (days) & $2.33 \pm 1.45$ & $2.94 \pm 1.29$ & 0.23 \\
\hline Mean time to return to normal activity (days) & $16.2 \pm 3.49$ & $15.9 \pm 4.31$ & 0.85 \\
\hline $\begin{array}{l}\text { Outcome of ERCP } \\
\text { Success rate (\%) }\end{array}$ & $90 \%$ & $93.75 \%$ & 0.67 \\
\hline Complications (n) & $3 / 30$ & $4 / 32$ & 1.000 \\
\hline Pancreatitis (n) & 2 patients & 2 patients & \\
\hline Hemorrhage (n) & 1 patients & 1 patient & \\
\hline Cholangitis (n) & 0 & 1 patient & \\
\hline Biliary stent (n) & 5 patients & 4 patients & \\
\hline Outcome of LC & & & \\
\hline Complications : & & & \\
\hline Infection (n) & 0 & 1 patient & \\
\hline Bleeding (n) & 0 & 0 & 1.000 \\
\hline Conversion(n) & $1 / 27$ & $2 / 30$ & \\
\hline Sub-hepatic drainage (n) & 3 patients & 4 patients & \\
\hline Mean patient satisfaction score ( /10) & $8.20 \pm 1.47$ & $8.44 \pm 1.50$ & 0.66 \\
\hline
\end{tabular}

hospital in the first post-operative morning in most cases. Group II Patients were given the choice to be discharged from hospital in the first post-ERCP morning in cases without complication and to be re hospitalized after 2 days for one day for LC or staying the whole period in hospital. Patient satisfaction score was designed by asking patients to express their satisfaction in a numerical score from 0 to 10 one month after surgery.

Patients were followed in visits at three day interval for two weeks then every 1 month for 6 months. Patients that did not come for follow up were contacted by telephone. Patients were encouraged to visit the clinic at any time if they have any problem.

Statistical analysis

Quantitative variables were expressed as mean \pm SD. Qualitative variables were expressed as frequency and percent. Quantitative parametric variables were compared between the two groups using the unpaired student t-test, quantitative nonparametric variables were compared using Mann-Whitney test. Qualitative variables were compared using Chi-square test or
Fisher exact test when the criteria for using Chi-square were not sufficient. The power used was 0.80 while the level of significance was $5 \%$.

\section{Results:}

Patients' characteristics: The characteristics of the two treatment groups, including baseline preoperative laboratory results are shown in Table (1). No significant differences were identified with respect to age, gender and other medical conditions.

Operation time: In group I, operative time ranged from 60-120 minutes (mean operative time: $97.3 \pm 17.9$ minutes). In group II, collective operative time ranged from 80-120 minutes (mean operative time: $104 \pm 13.6$ minutes). The difference between the two groups was statistically insignificant $(\mathrm{P}=0.27)$.

Hospitalization time: Hospitalization time ranged from 1-6 days in group I (mean hospitalization time: $2.33 \pm 1.45$ days). In group II, collective hospitalization time ranged from 2-6 days (mean hospitalization time: $2.94 \pm 1.29$ days). The difference 
between the two groups was statistically insignificant $(\mathrm{p}=0.23)$.

Time to return to normal activity: Time to return to normal activity ranged from 1021 days in group I (mean time: 16.2 \pm 3.49 days). In group II, time to return to normal activity ranged from 10-24 days (mean time: $15.9 \pm 4.31$ days). The difference between the two groups was statistically insignificant $(\mathrm{p}=0.85)$.

Level of direct bilirubin: Level of direct bilirubin returned to normal values in the blood 9 days after procedure in both groups Figure (1).

Outcome of endoscopic procedure: The success rate of endoscopic procedure was $90 \%$ for group I to $93.75 \%$ for group II. The difference was insignificant. (FET $\mathrm{p}=0.67$ ). Pancreatitis was observed in 2 patients in each group. Sphincterotomy related hemorrhage occurred in one patient in each group. One patient in group II experienced cholangitis. The total rates of endoscopic complications were $10 \%$ in group I and $12.5 \%$ in group II $($ FET $\mathrm{p}=1.000)$. Complications of ERCP were treated conservatively. Outcome of endoscopic procedure is shown in Table (2).

Outcome of laparoscopic cholecystectomy: No mortality was observed in both groups. Bile duct injury was not observed in this study. The overall LC related morbidity (including conversion) was $1 / 27$ in group I and 2/30 in group II (FET $p=1.000)$. Outcome of LC is shown in Table (2).

Patient satisfaction: Patients were asked to express their satisfaction in a numerical score from 0 to 10 one month after surgery. The mean patient satisfaction score for Group I patients was $8.20 \pm 1.47$ versus $8.44 \pm 1.50$ for Group II patients. The difference between the two groups was proved to be statistically insignificant $(\mathrm{p}=0.66)$.

Table (2), Comparison of operation time, length of hospital stay, time to return to normal activity and outcome of endoscopic and laparoscopic procedures between both groups.

\section{Discussion:}

Biliary surgeons are required to master more techniques than ever in the current era of minimal invasion for patients with concomitant cholodocolithiasis and cholecystolithiasis. Following the development of endoscopic techniques over the last three decades, these techniques have been confirmed as efficient and safe in the treatment of CBD stones. However, the diagnostic value of these methods in biliary diseases, especially benign diseases was markedly superseded by magnetic resonance cholangio pancreatography which has gradually become a better alternative noninvasive diagnostic technique in biliary diseases. ${ }^{13-15}$

ERCP combined with LC is widely used to treat concomitant cholodocolithiasis and cholecystolithiasis. However, ERCP and LC are often performed in two separate sessions. In this prospective randomized study, ERCP and LC were performed on the same session. We compared this method with the current practice where LC is performed after endoscopic stone extraction. Therefore, the outcomes are more convincing and objective than many other reports. ${ }^{12,16}$

Although this protocol was proposed years ago, only few medical faculties have carried out this procedure. A likely reason for this is that there is no specific standard process to refer to. In addition, the technique is difficult, especially when choosing the patient position in ERCP and managing distension of the intestine due to air insufflation.

Patients may be placed in the supine position. However, three problems can be faced; difficulty in locating the endoscopic tip in the second portion of the duodenum, trouble with positioning the papilla correctly and repeated interference due to liquid in the duodenal cavity. The disadvantages of the supine position may not be easily resolved due to the inherent anatomy of the papilla, which is located in the posterior medial aspect of duodenal wall. ${ }^{16,17}$

In comparison with the supine position, the prone position is optimal for cannulation of the papilla and obtaining good quality radiographic images. The prone position provides a natural pressure due to the weight 
of the abdomen, restricting distension of the intestine. General anesthesia with tracheal intubation can reduce patients' discomfort and make airway management easier. The only problem with the prone position is taking operators a few minutes to change the patient's position twice. Insufflating the least amount of air during ERCP and suctioning of the duodenal and gastric cavity thoroughly at the end of ERCP prevents intestinal distention that can make LC difficult.

The safety of the combination of laparoscopic and endoscopic techniques during the same session is a focus of concern for surgeons. Morbidity and mortality in endoscopic and laparoscopic procedures are very low with skilled operators. In the present study, there were no differences in complication rates and patient recovery between the two treatment groups, which demonstrated that this combination was safe.

LC with endoscopic stone extraction during the same session could be considered a safe procedure as the rate of LC-related complications, including bile duct injury and conversion, was low in our trial. This was consistent with previous results which showed that the earlier LC is performed after the endoscopic procedure; the better the outcome will be. ${ }^{10}$

For single session surgery for patients with cholodocolithiasis and cholecystolithiasis, laparoscopic CBD exploration (LCBDE) is another good choice rather than the combination of duodenoscopy and laparoscopy. These techniques have similar primary ductal clearance rates and morbidity. ${ }^{18,19}$

Studies showed that LCBDE is more cost effective and beneficial in the preservation of papillary function than ERCP, although suffering due to T-tube placement is a major disadvantage. Difficulties in LCBDE include extraction of the CBD stone and suturing of the $\mathrm{CBD}$ incision. With regard to the indications for LCBDE and endoscopic stone extraction, a clear consensus has emerged although different surgeons have different opinions. A CBD with a diameter smaller than $8 \mathrm{~mm}$ is regarded as a contraindication to LCBDE. 20,21

ERCP may not suite patients with large stones. Although endoscopic extraction of large stones has been reported, LCBDE is preferred in patients with stone diameter of $15 \mathrm{~mm}$ or greater. Laser lithotripsy by choledochoscope in LCBDE may be more direct and easier to manage large stones. For patients who are fit for both procedures, the choice involves patients' desire, hospital resources, and surgeon's ability. The longterm hazards of endoscopic sphincterotomy include recurrent stones, cholangitis, and cholangiocarcinoma, however, current evidence may partly dispel these associations. ${ }^{18,19}$

\section{Conclusion:}

Endoscopic stone extraction and LC performed during the same session is feasible, safe and effective alternative to two-stage ERCP and LC for concurrent cholodocolithiasis and cholecystolithiasis. It has many advantages including avoiding a second procedure without increasing the length of operation, hospital stay or conversion rates to open procedure. We recommend more studies to be done on this subject before its routine recommendation in surgical practice.

\section{Reference:}

1- Greca GL, Barbagallo F, Sofia M, Latteri S, Russello D: Simultaneous laparoendoscopic rendezvous for the treatment of cholecystocholedocholithiasis. Surg Endosc 2010; 24: 769-780.

2- Costi R, Mazzeo A, Tartamella F, Manceau C, Vacher B, ValverdeA: Cholecystocholedocholithiasis: A casecontrol study comparing the short- and long-term outcomes for a "laparoscopy first" attitude with the outcome for sequential treatment (systematic endoscopic sphincterotomy followed by laparoscopic cholecystectomy). Surg Endosc 2010; 24: 51-62.

3- Martin DJ, Vernon DR, Toouli J: Surgical versus endoscopic treatment of bile duct stones. Cochrane Database Syst Rev 2006; 2: CD003327.

4- Scientific Committee of the European 
Association for Endoscopic Surgery (E.A.E.S.): Diagnosis and treatment of common bile duct stones (CBDS): Results of a consensus development conference. Surg Endosc 1998; 12: 856-864 .

5- Phillips EH, Toouli J, Pitt HA, Soper NJ: Treatment of common bile duct stones discovered during cholecystectomy. $J$ Gastrointest Surg 2008; 12: 624-628 .

6- Bansal VK, Misra MC, Garg P, Prabhu M: A prospective randomized trial comparing twostage versus single-stage management of patients with gallstone disease and common bile duct stones. Surg Endosc 2010; 24: 1986-1989.

7- Lacitignola S, Minardi M: Management of common bile duct stones: A ten-year experience at a tertiary care center. JSLS 2008; 12: 62-65.

8- Schiphorst AH, Besselink MG, Boerma $\mathrm{D}$, Timmer R, Wiezer MJ, van Erpecum KJ, Broeders IA, van Ramshorst B: Timing of cholecystectomy after endoscopic sphincterotomy for common bile duct stones. Surg Endosc 2008; 22: 2046-2050.

9- Salman B, Yilmaz U, Kerem M, Bedirli A, Sare M, Sakrak O, Tatlicioglu E: The timing of laparoscopic cholecystectomy after endoscopic retrograde cholangiopancreaticography in cholelithiasis coexisting with choledocholithiasis. $J$ Hepatobiliary Pancreat Surg 2009; 16: 832-836.

10- Zang J, Zhang C, Zhou H, Gao J: Early laparoscopic cholecystectomy after endoscopic common bile duct stone extraction: The experience from a developing country. Surg Laparosc Endosc Percutan Tech 2011; 21: 120-122.

11- Cuschieri A, Lezoche E, Morino M, Croce E, Lacy A, Toouli J, Faggioni A, Ribeiro VM, Jakimowicz J, Visa J, Hanna GB: Multicenter prospective randomized trial comparing two stage vs single-stage management of patients with gallstone disease and ductal calculi. Surg Endosc 1999; 13: 952-957 .

12- Rogers S, Cello JP, Horn JK, Siperstein A, Campbell A, Mackersie R: Randomized controlled clinical trial of laproscopic cholecystectomy plus laparoscopic common bile duct exploration (LC+LCBDE) vs ERCP sphincterotomy plus laparoscopic Cholecystectomy (ERCP/S+LC)forcommon bile duct stone disease. J Gastroenterol
Hepatol 1999; 14(Suppl) :S110.

13- Maccioni F, Martinelli M, Al Ansari N, Kagarmanova A, De Marco V, Zippi M, Marini M: Magnetic resonance cholangiography: past, present and future: A review. Eur Rev Med Pharmacol Sci 2010; 14: 721-725.

14- Miao L, Fan ZN, Ji GZ, Wen W, Wang $\mathrm{X}$, Xiong GY, Jiang GB, Wang $\mathrm{M}$, Liu Z, Wu P: Comparative study of ultrasonography, magnetic resonance cholangiopancreatography and endoscopic retrograde cholangiopancreatography in common duct stones. Zhonghua Wai Ke Zazhi 2008; 46: 1465-1467.

15- Hekimoglu K, Ustundag Y, Dusak A, Erdem Z, Karademir B, Aydemir S, Gundogdu S: MRCP vs. ERCP in the evaluation of biliary pathologies: Review of current literature. $J$ Dig Dis 2008; 9: 162-169.

16- ElGeidie AA, ElEbidy GK, Naeem YM: Preoperative versus intraoperative endoscopic sphincterotomy for management of common bile duct stones. Surg Endosc 2011; 25: 1230-1237.

17- Terruzzi V, Radaelli F, Meucci G, Minoli G: Is the supine position as safe and effective as the prone position for endoscopic retrograde cholangiopancreatography? A prospective randomized study. Endoscopy 2005; 37: 1211-1214 .

18- Saito M, Tsuyuguchi T, Yamaguchi $\mathrm{T}$, Ishihara $\mathrm{T}$, Saisho $\mathrm{H}$ : Long-term outcome of endoscopic papillotomy for choledocholithiasis with cholecystolithiasis. Gastrointest Endosc 2000; 51: 540-545 .

19- Folkers MT, Disario JA, Adler DG: Longterm complications of endoscopic biliary sphincterotomy for choledocholithiasis: A North-American perspective. Am J Gastroenterol 2009; 104: 2868-2869.

20- La Greca G, Barbagallo F, Di Blasi M, Chisari A, Lombardo R, Bonaccorso R, Latteri S, Di Stefano A, Russello D: Laparoendoscopic "rendezvous" to treat cholecysto-choledocolithiasis: effective, safe and simplifies the endoscopist's work. World J Gastroenterol 2008; 14: 2844-2850 .

21- Tekin A, Ogetman Z, Altunel E: Laparoendoscopic "rendezvous" versus laparoscopic antegrade sphincterotomy for choledocholithiasis. Surgery 2008; 144: 442-447. 